\title{
Diverse cooling schedules to improved physico-mechanical properties of hot upset forged AISI 8620 PM steel bars
}

\author{
Y. G. Bala*, S. Raman Sankaranarayanan, K. S. Pandey \\ Department of Metallurgical and Materials Engineering, National Institute of Technology, \\ Tiruchirappalli 620 015, T. N., India
}

Received 3 September 2015, received in revised form 27 October 2015, accepted 28 October 2015

\begin{abstract}
The permanence of powder preform forged steels on higher order applications such as automobile, aerospace, ordnance industries with elevated temperature and strength is being sustained by implementing various heat treatment progresses. Different heat treatment schedules after the forged preforms of AISI 8620 steel produced through conventional powder metallurgy route were analysed for its mechanical strength, fractography, and microstructure properties. Green compacts were prepared in the pressure range of $540 \pm 10 \mathrm{MPa}$ and sintered in the electrical muffle furnace at $1150 \pm 10^{\circ} \mathrm{C}$ for $120 \mathrm{~min}$. Sintered compacts were hot upset forged into square cross section bars. Some bars were quenched in the water medium, and other sets of samples were cooled inside the furnace and in still air. Kinetics of the cooling velocities highly influences the tensile and impact properties improvement with determined microstructure and fractographic features.
\end{abstract}

K e y w o r d s: perform, forged, heat treatment, property, quenching, testing

\section{Introduction}

Excellent properties of the materials can be achieved with a modest modification of the technologies to sustain the material utilization for the long run costeffective process. Adopting the newer manufacturing technology and adding the process of heat treatments advances the material properties. Powder preform forging is the most eminent primeval and accepted worldwide green technology in attaining the superior mechanical properties of the materials. Powder forging is the process of deforming the sintered preforms developed by conventional powder metallurgy route in hot solid state condition either in the confined dies, open dies or partially open dies [1]. Although powder metallurgy covers very diversified domains of modern industry $[2,3]$, their development continuously stimulates the research effort for developing novel materials for advanced systems for long run reliability in critical applications. Further, the pores and voids in sintered preforms suppress the ability to attain the full density to the preforms required to reach the probable mechanical properties for higher applications. To prevail these hinders, the powder forging combining the technology of powder metallurgy and forging process has proved effective method to improve the density and strength of sintered parts [4] as the densification obviously improves the properties of the materials suitable for the higher order applications with lack of any localized porosity $[5,6]$. The majority of the engineering components can be produced to their theoretical density by sintering followed by forging [7], and their properties are comparable to those of conventional wrought steel and with possible competitive processes that are suitable for structural and automotive applications of their enhanced tensile strength [8, 9]. Apart from the powder forging process, sound metallurgical properties can be obtained by adding the heat treatment processes. Generally, the heat treatments are applied to any material to modify its metallurgical structure and alter its physical, mechanical, and chemical properties. In addition, the treatment uses phase transformation during heating and cooling to change a microstructure in a solid state, which is part of the materials to substantiate its strength. End users of the greatly stressed part have expressed a preference for

*Corresponding author: e-mail address: ygbalasingam@yahoo.com 
Ta ble 1. Powder properties of AISI 8620 steel composition

\begin{tabular}{clc}
\hline Sl. No. & Property & AISI 8620 \\
\hline 1 & Flow rate by hall flowmeter (s/50 g) & 22.93 \\
2 & Apparent density (g/cc) & 3.399 \\
3 & Tap density & 5.523 \\
\hline
\end{tabular}

low alloy steels such as AISI 41XX, AISI 43XX, and AISI 86XX. More efficient alloying elements enable a fully martensitic microstructure attained at the lower quench rate in a larger size with low carbon content, reducing the risk of cracking during part production and service [10]. Low alloy steel constituting nickelchrome-molybdenum as the alloying elements exhibits superior mechanical properties comparatively and increases the hardenability, strength, and toughness after heat treatment [11]. The present investigation also aims to examine a similar category of low alloy nickelchrome-molybdenum steel of AISI 8620 steel, which is popularly used in many industries due to its relatively low cost and high quality, used in demanding environments where solutions better than carbon steel are required. Alloy 8620 is a low carbon case hardening alloy that features a hard case with a tough, ductile core which experiences minimal distortion during heat treatment. This alloy steel provides a combination of toughness, hardness, strength and impact performance not found in lower grade carbon steels. This alloy is typically used for carburized parts where a hardened case is desired for wear resistance. This grade finds wide application in the automotive industry and for ring gears, pinions, racks and worms, helical gears, bearing races, pins, and high tensile chains [12]. The importance of the material applications and the processing technology of powder forging combined with general heat treatment process always reflect on the continual endeavors.

\section{Experimental details}

\subsection{Materials required}

The materials required to conduct the present experimental work are the elemental powders procured from standard metal powder companies taken in weight percentage ratio of order $97.47 \% \mathrm{Fe}, 0.20 \%$ C, $0.28 \% \mathrm{Si}, 0.80 \% \mathrm{Mn}, 0.55 \% \mathrm{Ni}, 0.50 \% \mathrm{Cr}$, and $0.20 \%$ Mo. Stainless steel pots with tighten lids and ceramic balls were required for blending the powder mixes. High Carbon High Chromium die with the suitable bottom insert and punch set up and 1.0 MN capacity universal testing machine were used to consolidate the homogeneously blended powder into solid green compacts. The graphite mixed acetone paste was used to lubricate the punch and die to avoid the friction and poor surface finish during consolidation and proper ejection of the green preforms. The thin layer of indigenously developed ceramic coating was applied all over the compact surface and allowed to dry at room temperature. Electrical muffle furnace capable of maintaining the temperature of $1150 \pm 10^{\circ} \mathrm{C}$ and suitable thermocouple for measuring the temperature were required. A friction screw press of 1.0 MN capacity to hot upset forging sintered preforms, and different quenching media like water and air were required to quench the forged preforms and cool down to the room temperature. Apart from this, a standard hall flowmeter to measure the powder properties, a Hounsfield tensometer to conduct the tensile test according to the standard ASTM E8/E8M-13, and Izod impact testing machine to measure the impact strength according to the standard ASTM D256 were used, as well as an optical microscope to evaluate the microstructure of the forged preforms and a scanning electron microscope to reveal the fractography of the fractured samples.

\subsection{Preparation of preforms}

The required elemental powders were taken according to the weight percentage and blended in the tight sealed stainless steel pot mill along with the ceramic balls with the powder to ball ratio $1: 1$ for the period of $37 \mathrm{~h}$ until the homogeneous mixture of the blend was obtained. The homogeneity of the blend was validated by measuring the standard powder properties like apparent density, tap density, and flow rate using the standard hall flowmeter. For every one hour of blending, a $100 \mathrm{~g}$ of powder were taken out from the pot mill and examined for the above said properties. For the consecutive reading where the measurements are being constant or repeated, it has been evident to attain the homogeneous powder blend, and these properties are shown in Table 1. Further, the composition of the blend was validated for its quantitative analysis and morphology by scanning electron microscopy shown in Figs. 1a,b. The pre-weighed homogeneously blended powder was consolidated into the green compact using the suitably lubricated die and punch setup in Fig. 2a using 1.0 MN capacity universal testing machine in the pressure range of $540 \pm 10 \mathrm{MPa}$ with initial preform density equivalent 
to $86 \pm 1 \%$ of the theoretical density. Further, the compact green surfaces coated with the thin layer indigenously developed ceramic coating two times perpendicular to the surface to first coating. This coating is essential to protect the preform against the oxidation during sintering and forging. The coated compacts were sintered inside the electrical muffle furnace $1150 \pm 10^{\circ} \mathrm{C}$ and maintained for the period of $120 \mathrm{~min}$ as the sintering time. The sintered preforms were hot upset forged into square cross section bars of $120 \mathrm{~mm} \times 10 \mathrm{~mm} \times 10 \mathrm{~mm}$ using $1.0 \mathrm{MN}$ capacity hydraulic friction screw press. The overall methodology executed is shown in Fig. $2 \mathrm{~b}$.

\subsection{Heat treatments and testing}

A set of twelve sintered preforms were hot upset forged to square cross section bars, and each four sets of samples out of these twelve square bars were given different cooling medium such as sintered forged homogeneous water quenching (SFHWQ), sintered forged homogeneous furnace cooling (SFHFC), and sintered forged homogeneous air-cooling (SFHAC). The entire bar samples after cooling down to the room temperature were machined to the standard dimensions required to carry out the tensile and impact tests according to the standards mentioned at room temperature. However, the test results were analysed by averaging the test values of these four sets of samples in each heat treatment. Further, initial gauge length and initial diameter were measured before testing. Final gauge length and final diameter were measured after testing by combining the two fractured parts. Fracture load and maximum load were noted down from which the ultimate tensile strength, yield strength, percentage elongation, percentage area reduction including true and engineering fracture stress were calculated. All the testing and analysis have revealed that the quenching media and the different cooling velocities affect the properties domain distinctly.
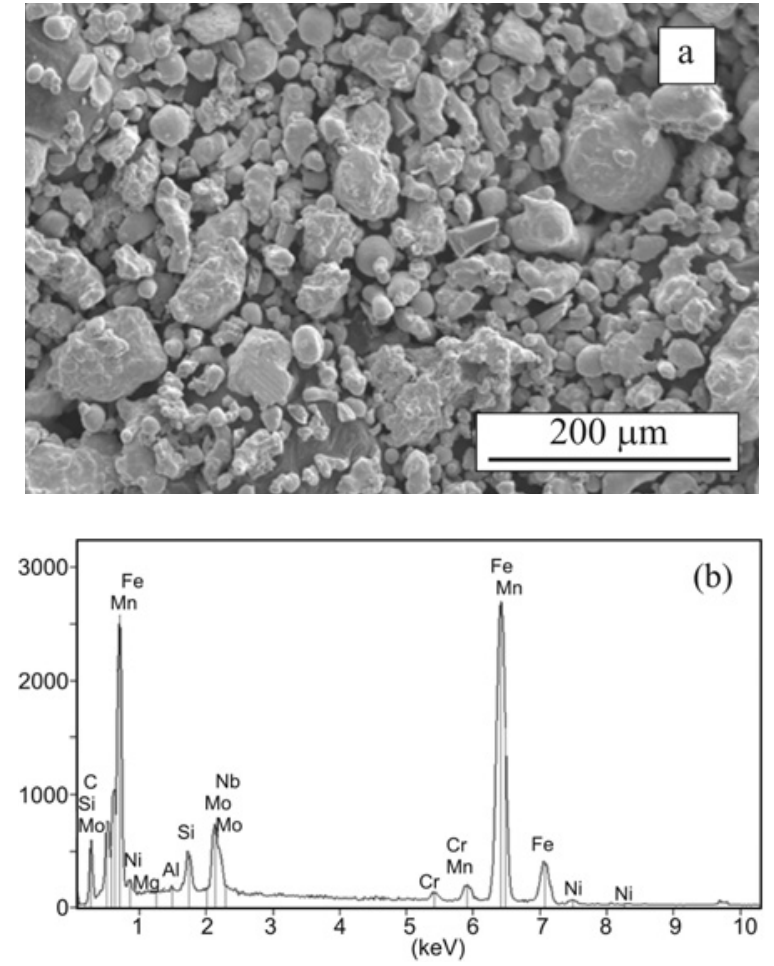

Fig. 1. Powder morphology of AISI 8620 after $37 \mathrm{~h}$ blending (a) and distribution of elemental powder in AISI 8620 powder blend (b).

\section{Results and discussion}

\subsection{Testing and mechanical properties}

The hot upset forged square cross section bars were machined according to the standard dimensions required to conduct the tensile test and impact test. The Hounsfield tensometer was used to conduct the tensile test, and Izod impact test machine was used to conduct the impact test without notch. The test results such as impact strength, yield strength, ulti-

(a)
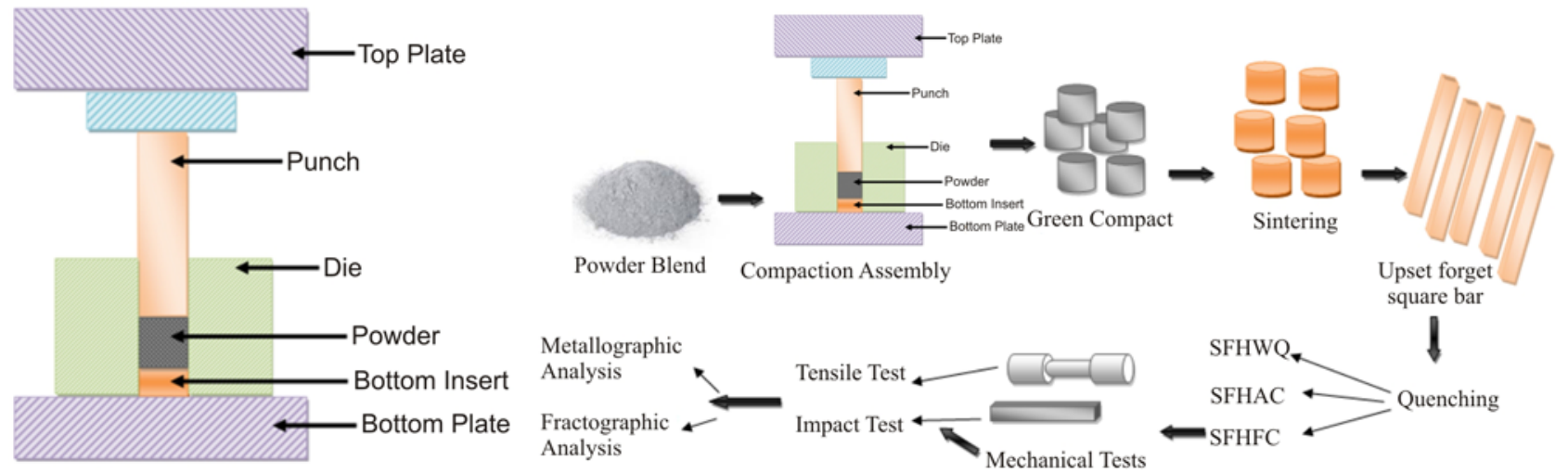

Fig. 2. Compaction assembly (a) and experimental procedure (b). 
Ta ble 2. Mechanical properties of AISI 8620 for different heat treatments

\begin{tabular}{|c|c|c|c|c|c|c|c|}
\hline \multirow[b]{2}{*}{$\begin{array}{c}\text { Heat } \\
\text { treatments }\end{array}$} & \multicolumn{6}{|c|}{ Tensile properties } & \multirow[b]{2}{*}{$\begin{array}{c}\text { Impact } \\
\text { strength } \\
(\mathrm{J})\end{array}$} \\
\hline & $\%$ Elongation & $\begin{array}{l}\% \text { Area } \\
\text { reduction }\end{array}$ & $\begin{array}{l}\text { True stress at } \\
\text { fracture } \\
(\mathrm{MPa})\end{array}$ & $\begin{array}{l}\text { Engg. stress } \\
\text { at fracture } \\
(\mathrm{MPa})\end{array}$ & $\begin{array}{l}\text { Yield stress } \\
\qquad(\mathrm{MPa})\end{array}$ & $\begin{array}{c}\text { Ultimate } \\
\text { tensile stress } \\
(\mathrm{MPa})\end{array}$ & \\
\hline SFHFC & 10.265 & 21.607 & 792.21 & 670.67 & 595.60 & 686.15 & 125 \\
\hline SFHAC & 8.567 & 15.353 & 939.36 & 736.50 & 679.66 & 752.98 & 156 \\
\hline SFHWQ & 1.135 & 5.555 & 1510.57 & 1333.66 & 710.18 & 1335.50 & 56 \\
\hline
\end{tabular}
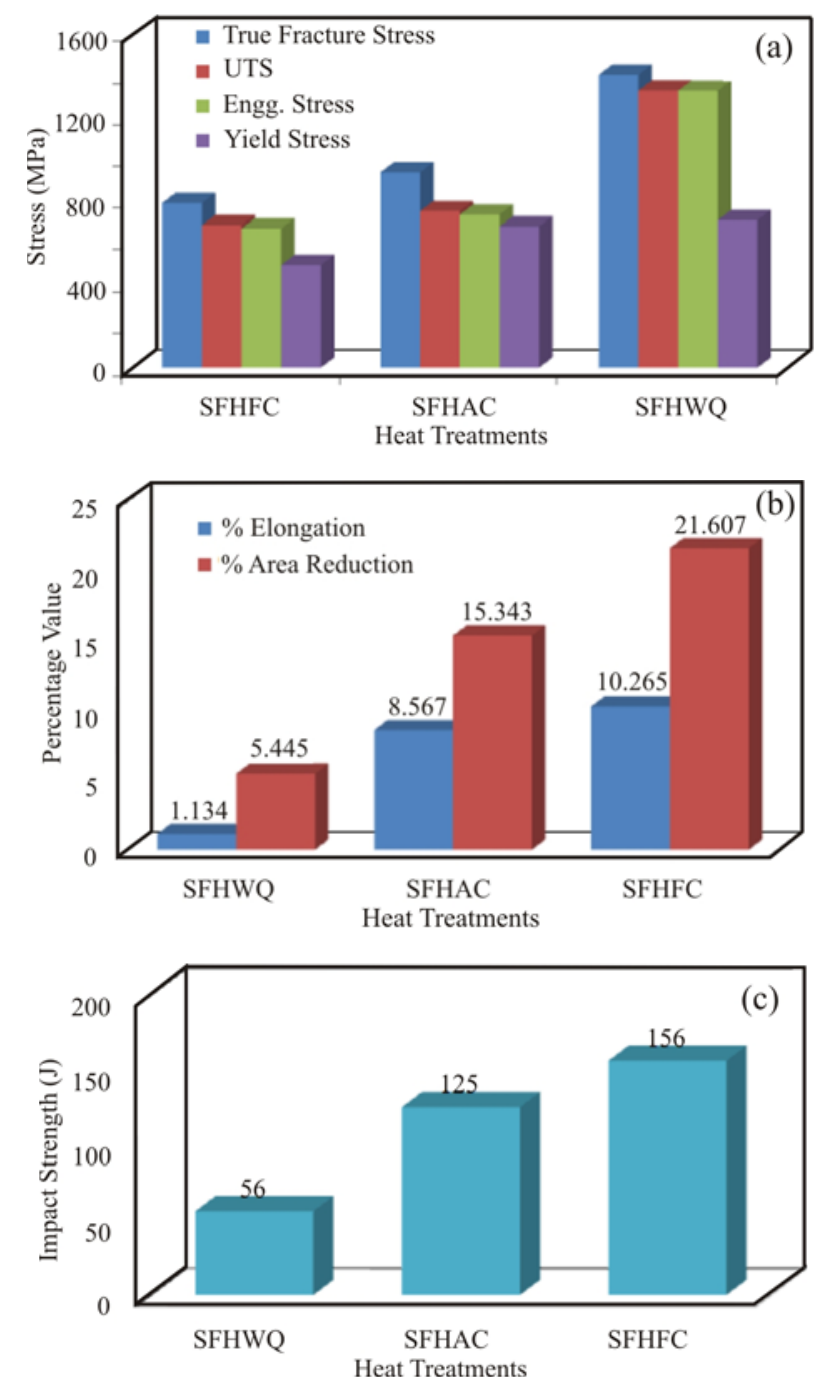

Fig. 3. Bar chart showing (a) the tensile properties for various heat treatments, (b) the $\%$ elongation and $\%$ area reduction for various heat treatments, and (c) the impact strength for various heat treatments.

mate tensile strength, true and engineering fracture stress, along with percentage elongation and percentage area reduction are tabulated in Table 2 . The distinguished comparisons of the properties are shown
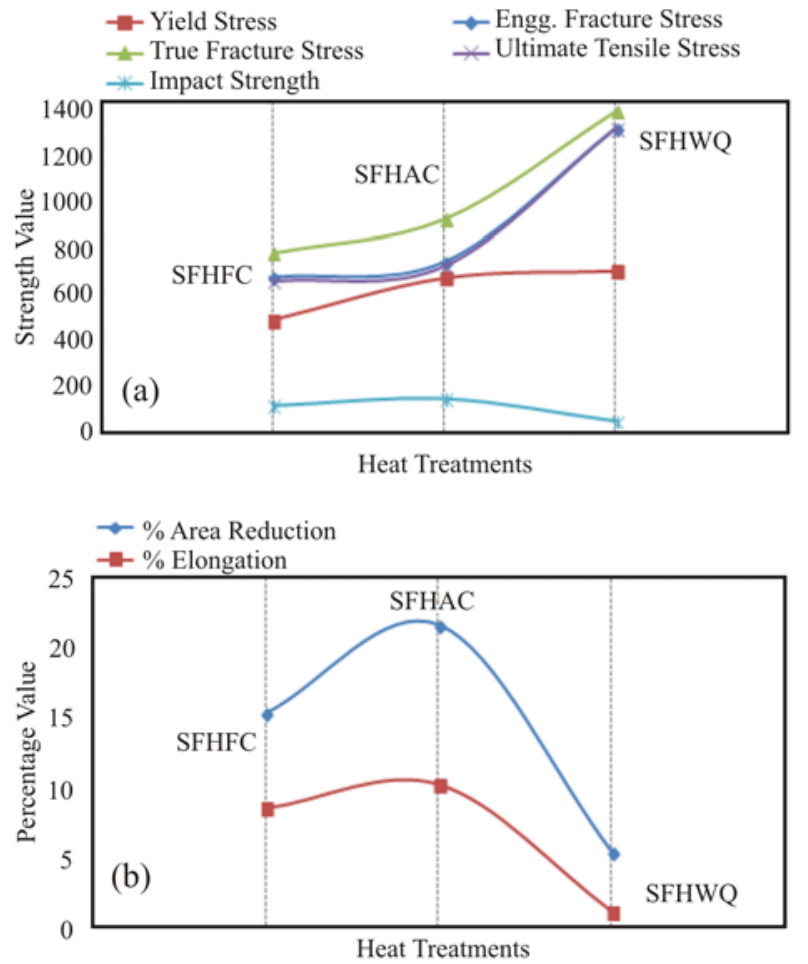

Fig. 4. Line chart of (a) tensile properties for various heat treatments (b) \% elongation and \% area reduction for various heat treatments.

by the bar chart in Figs. 3a,b,c, and line charts shown in Figs. 4a,b represent the tendency of tensile properties with respect to the cooling medium. The mechanical properties of AISI 8620 steel developed through hot upset powder preform forging route under different cooling kinetics are listed in Table 2. Figures 3 and 4 show that the properties are greatly influenced and improved by varying the rate of cooling. Figure 3a represents the influence of tensile strength such as yield strength, ultimate tensile strength, true and engineering fracture stress for the heat treatment of SFHFC, SFHAC, and SFHWQ. Figure 3b represents the influence of percentage elongation and percentage area reduction. Figure $3 \mathrm{c}$ shows the influence of impact strength for the same order of quench media. 
Based on the tested values and the charts, the rate of the cooling process highly depends upon the quenching medium which instigates the material properties of the system. The rapid rate of cooling imparts the higher tensile strength, and the slower cooling rate imparts the improved toughness of the material. Hence, observing the properties and the medium of cooling, the rate of cooling is superior in the water, followed by the air and furnace. Table 2 and the comparison chart in Fig. 3a also disclose that the water quenched sample has maximum yield and ultimate tensile strength. In addition, the true and engineering fracture stresses retain in the same manner. Whereas, the order of yield strength, ultimate tensile strength, and fracture next inferior to the sinter forged homogenized water quench sample is the sinter forged homogenized air cooled sample and the least is the sinter forged homogenized furnace cooled sample as in Fig. 3a. Comparing the percentage elongation and percentage area reduction for the above said heat treatment processes with respect to Fig. 3b, it is in the vice versa, that is, the percentage elongation and percentage area reduction were superior for the furnace-cooled sample followed by the air cooled and least for the water cooled sample. This also shows that the evidence of the ductility and brittleness built up in the material varies with the cooling velocity. Though the sinter forged homogenized water quenched sample shows superior in the tensile strength, the failure of the sample is by brittleness which is obvious by its toughness value resulted in the impact test was inferior when compared to other heat treated samples, as also influenced by the velocity of cooling or heat treatment steps. However, the toughness was highly developed in sinter forged homogenized furnace cooled followed by sinter forged homogenized air cooled sample as shown in Table 2 and Fig. 3c. Further, the mode of fracture for air cooled and furnace cooled samples are influenced by the ductility, which is evident, by its toughness value and percentage of elongation and subsequent area reduction listed in Table 2. In addition, comparing the trend of the tensile strength such as yield strength, ultimate tensile strength, true and engineering fracture stress with respect to percentage elongation and per area reduction by the line chart as shown in Figs. 4a and $4 \mathrm{~b}$ for three different heat treatments reflects that the increasing order of the strength shows the reducing value of the percentage of elongation and percentage area reduction. Tensile property strength for the water quenched sample resulted in the higher value of the curves for the least percentage of elongation and area reduction. The minimum values of the tensile property for sinter forged homogenized furnace cooled sample show the maximum percentage of elongation and area reduction, and subsequently for sinter forged homogenized air cooled sample. The data points of the test values of the ultimate tensile strength and the engineering strength at fracture almost coincide for the respective heat treatments in the same manner discussed earlier. There is a similar pattern of curve trending for the impact strength and percentage elongation and percentage area reduction for the heat treatments mentioned for the toughness.

\subsection{Microstructure}

The mechanical properties developed in any of the material systems are also warranted by the marked microstructure induced through the process. Mechanical properties of steels are strongly associated with their microstructure obtained after heat treatment processes. Figures 4a,b,c show the microstructure of AISI 8620 sintered forged for differently heat-treated sample revealed by etching $2 \%$ Nital and imaged on $400 \times$ magnification using optical microscopy. Figure $4 \mathrm{a}$ corresponds to the microstructure of sintered forged homogenized furnace cooled, Fig. 4b represents the microstructure of sintered forged homogenized air cooled and Fig. 4c shows the microstructure of sintered forged homogenized water quenched sample. An analysis of the features of microstructure for these heat treatment processes, it shows the noticeable relationship with respect to the mechanical properties. The microstructure of the sintered forged homogenized furnace cooled sample shown in Fig. 4a illustrates the foremost full whitish coarse ferrite structure and hardly few pale blackish pearlites with large sized grains. This provides the information that the sintered forged homogenized furnace cooled sample is soft and ductile in nature and has good impact strength. This is obvious in slower cooling rate. The microstructure for the sintered forged homogenized air cooled sample in Fig. 4b shows the irregular austenite structure with grain boundaries. This implies that the air medium has contributed to improving the mechanical properties of grain homogeneity to the modest level of restrained toughness. Figure 4c shows the microstructure for the sintered forged homogenized water quenched sample results in the martensitic phase structure, which is a very hard and brittle as noticeable in faster cooling rate. The above discussions were justified as the nature of the microstructure revealed for the composition AISI 8620 matches with the mechanical properties analysed in the order of heat treatment discussed above.

\subsection{Fractography}

The fractured surface of the AISI 8620 steel composition developed through hot upset powder preform forged and differently heat treated sample was observed by scanning electron microscopy under $200 \mathrm{mi}-$ crons magnification. Figure 5a shows the fractographic surface of the sintered forged homogenized furnace 

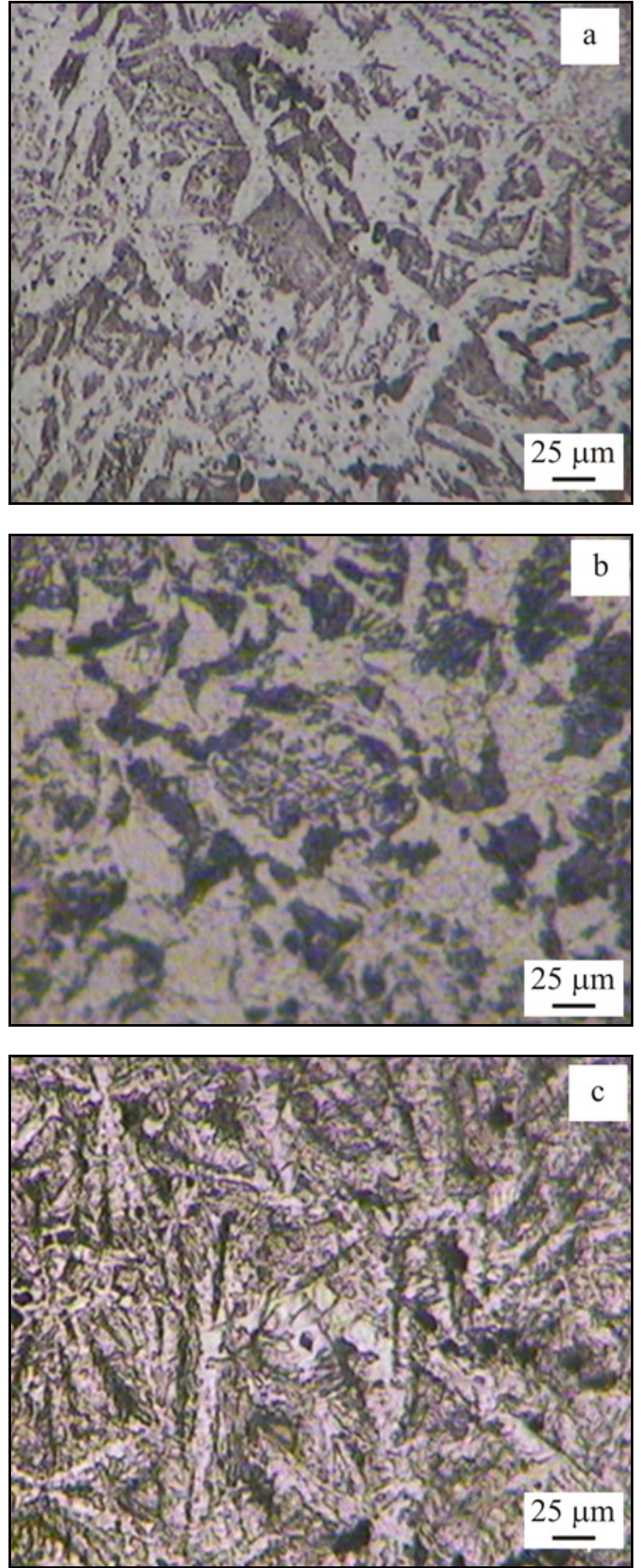

Fig. 5. Microstructures for (a) SFHFC, (b) SFHAC, and (c) SFHWQ.

cooled, Fig. 5b represents the fractographic surface of the sintered forged homogenized air cooled and Fig. 5c represents the fractographic surface of the sintered forged homogenized water quenched sample. The furnace cooled sample and air cooled sample show the influence of ductile mode of fracture which very clearly shows uniformly distributed dimples markings with
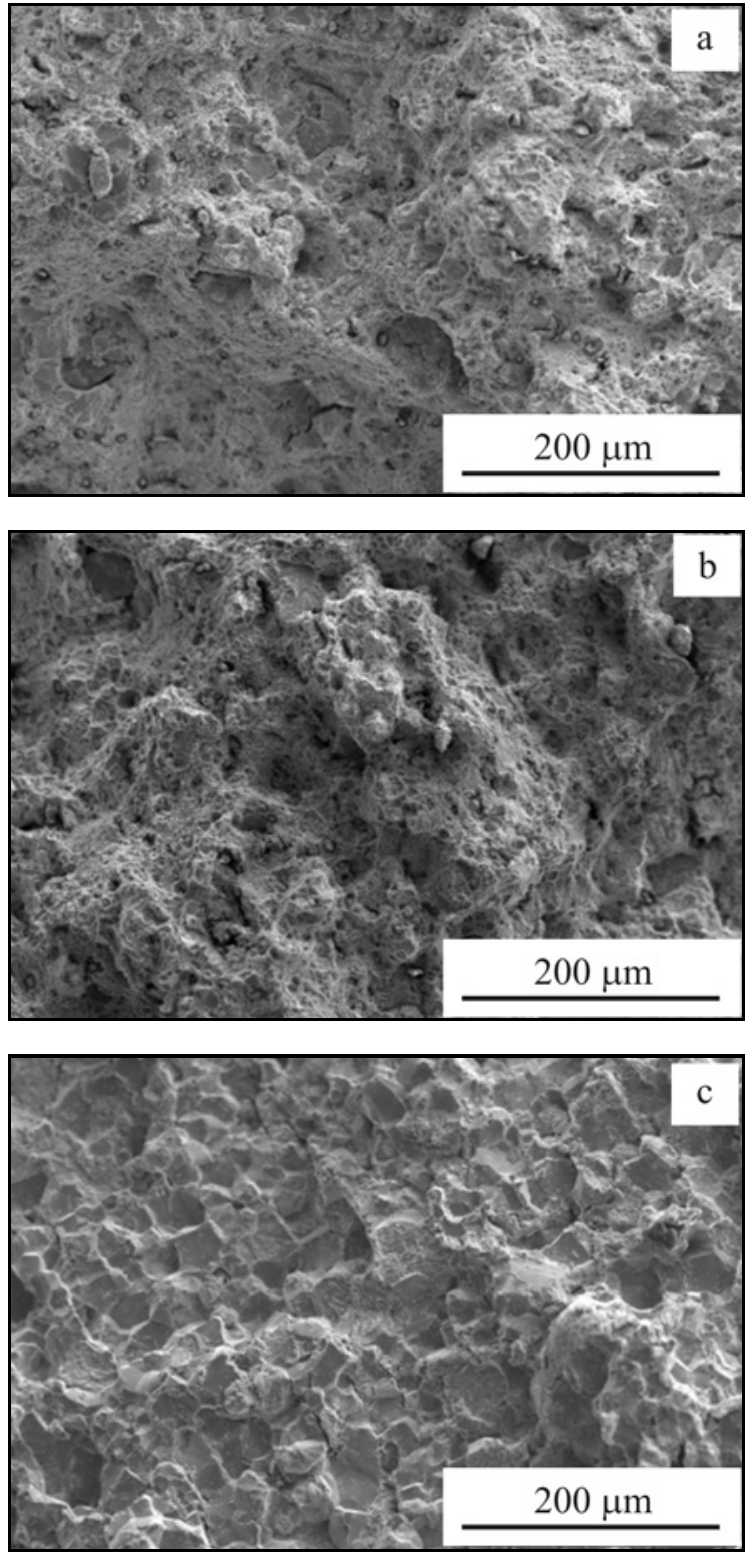

Fig. 6. Fractograph images for (a) SFHFC, (b) SFHAC, and (c) SFHWQ.

voids to initiate the crack and limited cleavage markings. The sintered forged homogenized water quenched sample in Fig. 5c presents the fracture cleavage surface with a diminutive amount of dimples showing that the transgranular and intergranular cleavage mode of fracture is caused generally by brittleness.

\section{Conclusions}

Based on the critical analysis of experimental data and mechanical testing of sintered forged AISI 8620 steel composition developed through hot upset forged and differently heat treated samples for mechanical 
properties, microstructure and fractographic analysis, main conclusions are listed underneath:

1. Values of mechanical properties like tensile and impact fracture strength are found to improve with a different mode of homogenized heat treatment. It has resulted that the tensile strength is superior in SFHWC followed by SFHAC and SFHFC. Impact strength raised in order of heat treatment of SFHFC, SFHAC, and least in SFHWQ.

2. The influence of ductility and brittleness as the function of percentage elongation and percentage area reduction is greatly induced by the virtue of the rate of cooling. The degree of slow cooling in SFHFC and SFHAC shows the significant amount of ductility, whereas, the fastest cooling in SFHWQ shows the noticeable brittleness.

3. Fractography of the fractured surface has been established in a mixed mode of failure, i.e. mostly brittle and partly ductile in SFHWQ and mostly ductile and partly brittle in the case of SFHFC and SFHAC.

4. It is categorically established that by using elemental powders, it is possible to produce highly dense products by monitoring blending, compaction, sinter forging and by controlling the heat-treating schedules appropriately.

Thus, the present investigation of this paper finally concludes that the superior properties demanded by the automotive and structural parts for the steels by the industry and the other applications could gain the possibility of needs by developing the research attention toward the powder forging technique.

\section{References}

[1] James, W. B., McDermott, M. J., Powell, R. A.: Metals Handbook 14. 9th Edition. Materials Park, ASM International 1988.

[2] Orban, R. L.: Romanian Reports in Physics, 56, 2004, p. 505. http://www.rrp.infim.ro/2004_56_3/RLOrban.pdf

[3] Slattery, R., Hanejko, F. G., Rawlings, A. J., Marucci, M., Narasimhan, K. S.: Gear Technology, 21, 2005, p. 39.

[4] Wang, G., Zhao, G.: Journal of Material Science and Technology, 18, 2002, p. 544.

[5] Grady, T. G.: Journal of Material Shaping Technology, 7, 1989, p. 105. doi:10.1007/BF02833776

[6] Bockstiegel, G., Blande, C. S.: Powder Metallurgy International, 8, 1976, p. 155.

[7] Pandey, K. S.: Key Engineering Materials, 29-31, 1989, p. 465. doi:10.4028/www.scientific.net/KEM.29-31.465

[8] Shanmugasundaram, D., Chandramouli, R.: Materials and Design, 30, 2009, p. 3555. doi:10.1016/i.matdes.2009.03.020c

[9] Brown, G. T., Steed, J. A.: Powder Metall., 17, 1974, p. 157. doi:10.1179/pom.1974.17.33.011

[10] Causton, R. J., Cimino, T. M.: In: Proceedings of International Conference and Exhibition on Powder Metallurgy and Particulate Materials. Eds.: Chaman, L., Neupaver, A. J. Princeton, Metal Powder Industries Federation 1994, p. 1. http://www.gkn.com/ hoeganaes/media/Tech\%20Library/22.\%20High\% 20Density\%20Processing\%20of\%20Cr-Mn\%20PM\% 20Steels.pdf

[11] Totten, G. E.: Steel Heat Treatment Metallurgy and Technologies. 2nd Edition. Boca Raton, CRC Press 2007.

[12] http://www.amcastle.com/stock/alloy-steel/grade8620-uns-g86200-alloy-bar-tube-plate.aspx 